\title{
User Perception and Expectations on Deleting Instant Messages - or- "What Happens If I Press This Button?"
}

\author{
Theodor Schnitzler*, Christine Utz*, Florian M. Farke*, Christina Pöpper ${ }^{\dagger}$, and Markus Dürmuth* \\ ${ }^{*}$ Ruhr-Universität Bochum, Germany ${ }^{\dagger}$ New York University Abu Dhabi, United Arab Emirates \\ theodor.schnitzler@rub.de christine.utz@rub.de florian.farke@rub.de \\ christina.poepper@nyu.edu_markus.duermuth@rub.de
}

\begin{abstract}
Contemporary mobile messaging provides rich text and multimedia functionality leaving detailed trails of sensitive user information that can span long periods of time. Allowing users to manage the privacy implications both on the sender and the receiver side can help to increase confidence in the use of communication applications. Recently, in October 2017, one of the mobile messengers with the largest user base, WhatsApp, has introduced a feature to delete past messages from communication, both from the sender's and the receiver's logs.
\end{abstract}

In this paper, we report on a study with 125 participants conducted in a between-subjects design. We explore the actual demand for deleting mobile messages, and we investigate how well users comprehend this functionality as implemented in popular messaging applications. We found statistically significant differences in users' understanding of message deletion between our three test conditions, comprising WhatsApp, Facebook Messenger, and Skype. $80 \%$ of participants in the WhatsApp condition could correctly assess the effects of deleting messages, compared to only $49 \%$ in the Skype condition.

Our findings indicate that users demand a message deleting functionality and that they can more precisely estimate the capabilities of a deletion function when its effects are transparently explained in the application's user interface.

Keywords-usability, privacy, messaging, mobile systems, digital forgetting

\section{INTRODUCTION}

As Internet-connected smartphones are prevalent nowadays, instant messaging applications on these devices are very popular, resulting in more and more people using mobile messaging apps in their daily communication with their peers [1]. In addition to one-to-one conversations, these apps facilitate group chats and support various message types such as text, picture, video, or voice messages.

In contrast to face-to-face talks or telephone calls, the course of a conversation in mobile messaging is usually logged by each participant. Logging makes the communication persistent and allows previously uninvolved third parties to retrieve past communication from the message history. Since

European Workshop on Usable Security (EuroUSEC) 2018

23 April 2018, London, England

ISBN 1-891562-54-1

https://dx.doi.org/10.14722/eurousec.2018.23009 communication in mobile messengers is often informal, it seems plausible that messages are often of ephemeral nature and not meant to be stored permanently.

Moreover, the increasing use of mobile messaging in everyday life carries the risk of accidentally sending messages to the wrong recipient. This can be a serious threat to users' privacy, especially when the communication contains sensitive personal information [2], [3]. Even if the availability of a proper revocation mechanism cannot completely eliminate these threats, the risks could at least be reduced when there is a chance to delete such a message before the recipient has read it. Related topics concerning the protection of personal privacy, most importantly the Right to be Forgotten, have found considerable attention over the last years, especially in Europe, resulting in the establishment of the General Data Protection Regulation (GDPR) [4], [5].

There are various reasons for users to delete messages described in the following. Users can freely decide to maintain their local message history, but also to delete specific messages from their own devices, e.g., to free memory on the device, or to clean up old conversations for clarity reasons. In contrast, deleting messages from the recipient's message history is typically a much more difficult issue, especially in decentralized, open systems such as e-mail or non-proprietary Jabber/XMPP instant messaging networks where users can run their own client and server software. However, the most popular mobile messaging apps are part of closed ecosystems, which are not designed for interoperability, and therefore most users rely on client software and servers provided by a particular vendor.

In October 2017, the messenger WhatsApp introduced a new feature which allows users to choose whether a sent message is to be deleted only locally or also from the recipient's conversation $\log$ [6], [7]. If users choose the latter, the message is replaced with a note indicating that the message has been deleted. This also applies to messages the recipient has already read. The release of the Deleting Messages for Everyone ${ }^{1}$ feature indicates that the actual effect of the deletion functionality had not been explicitly stated before, thus raising the question whether the effects of such functions are apparent to the users. Other popular mobile instant messaging applications such as Facebook Messenger and Skype present the functionality for deleting messages in a similar fashion but do not explicitly explain its different effects.

\footnotetext{
${ }^{1}$ https://blog.whatsapp.com/10000635/Deleting-Messages-for-Everyone
} 
To shed light on users' perception of message deletion, we conducted a user study to investigate whether the participants understand the actual functionality of message deletion in instant messaging applications. In particular, we explore the following research questions on user expectations towards these functions:

\section{RQ1 What are users' expectations towards the functionality} of deletion mechanisms?

RQ2 Do specific implementations of this functionality match users' perceptions, i.e., do users correctly estimate the consequences of a particular message deletion?

This is interesting because, right now, users are bound to choices that designers and developers have made-long before when initially building their applications. It is unclear to what extent actual users and their feedback were involved in the underlying decision processes. In order to make devices such as smartphones better agents for their users, the capabilities of applications need to fit the users' needs. In particular, users should not have to face surprises because an effect triggered by their action does not match what they expected the action to do. While our study explores users' needs in message applications, it can also provide valuable insights for developers to design features in their applications more comprehensible and usable. It has been well-known for almost two decades that failures in user interface design make it impossible for users to apply security features correctly [8]. fold:

Our major findings and contributions in this work are three-

1) We show that those participants of our study who have deleted messages had various reasons for demanding deletion of messages, ranging from textual improvements to withdrawing messages that have been sent mistakenly or that are considered inappropriate in retrospect.

2) Our results demonstrate a demand for an active selection on a per-message basis regarding where messages selected for deletion should indeed be deleted from (sender or recipients), as desired by more than $40 \%$ of our study participants.

3) Our results indicate that the participants can better assess the effects of deleting messages when the functionalities are explained transparently. We reveal that the example implementation of WhatsApp can help developers to improve the user experience of their applications.

\section{DELETING MESSAGES}

Mobile messaging, i.e., communication using mobile devices such as smartphones via apps such as WhatsApp, Facebook Messenger, or WeChat, has a large user base and is regularly used for personal communication with friends or family. Many of these apps offer the possibility to delete messages, while the concrete implementations widely differ between different apps.

We have investigated the characteristics of the implemented delete functionality for 12 popular messaging applications (cf. Table I). We selected apps with a high number of monthly active users [9], concentrating on apps whose primary focus is messaging, and additionally including messengers focused on protecting user privacy, such as Signal or Threema.

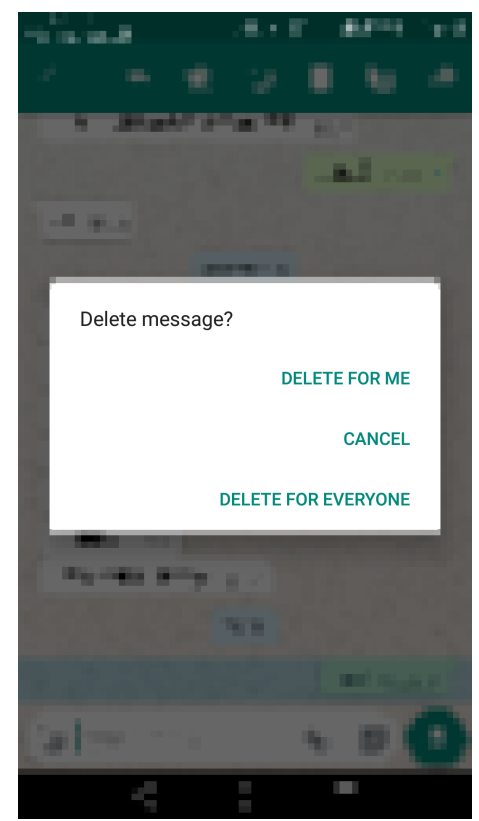

Fig. 1. Dialog for confirmation of deleting a message in WhatsApp. Users can choose whether the message should be deleted only from the sender's conversation log or also from the recipients' logs.

We identified several conceptual differences between the implementations, which we discuss in the remainder of this section.

\section{A. Local vs. Global Deletion}

The effects of deleting a message differ between applications. Except for Google Hangouts, all applications under consideration (Table I) support to Delete Messages Locally from the sender's message history. Hangouts differs in that it only allows to delete the entire conversation history with the respective contact.

The majority of applications also allow messages to be removed from the recipients' message histories, denoted Delete Message Globally. Popular applications supporting this feature include WhatsApp and Skype. We think the effects of the deleting mechanism of a given messenger are not always obvious. Whereas WhatsApp explicitly distinguishes between the options Delete for me and Delete for everyone (see Figure 1) in a prompt after the message has been selected to delete, Skype only provides one Delete function that removes the message from the message logs of all participants in the conversation. In both applications, deleting messages globally is time-limited. In WhatsApp, this option is available for seven minutes $^{2}$ after sending a message, Skype does not explicitly specify its limit. Viber provides a functionality identical to WhatsApp, having already introduced this feature in 2015 [14]. In WeChat, users can Recall their latest message within two minutes after sending [15]. However, this functionality is not integrated into the existing delete option but can be selected via an additional menu item. In December 2017, Line also introduced the Unsend option, which allows users within a

\footnotetext{
${ }^{2}$ As of 6 March 2018, WhatsApp has announced to extend this time limit to 68 minutes and 16 seconds [13] in future versions.
} 
TABLE I. DELETING MESSAGES IN MOBILE INSTANT MESSAGE APPLICATIONS. THE NUMBERS OF MONTHLY ACTIVE USERS ARE LiSTED IN MILLIONS. A FILLED CIRCLE ( ) INDICATES THAT THE APPLICATION PROVIDES THE RESPECTIVE PROPERTY, AN EMPTY CIRCLE (O) DENOTES THAT THE PROPERTY IS NOT PROVIDED. IF A CERTAIN PROPERTY DOES NOT APPLY TO A PARTICULAR APPLICATION, WE MARK THIS WITH A DASH (-). FOR EXAMPLE, SEPARATE DELETION FUNCTIONALITY ONLY APPLIES IF THE MESSENGER SUPPORTS BOTH TYPES OF DELETING MESSAGES (LOCALLY AND

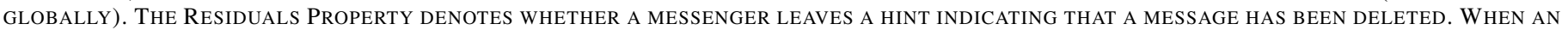
APPLICATION SUPPORTS MESSAGES THAT AUTOMATICALLY DISAPPEAR, THIS IS DENOTED EPHEMERAL MESSAGES. ALL PROPERTIES APPLY TO THE LATEST AVAILABLE APPLICATION VERSIONS, AS OF FEBRUARY 2018.

\begin{tabular}{|c|c|c|c|c|c|c|c|}
\hline Messenger & $\begin{array}{l}\text { Monthly } \\
\text { Active } \\
\text { Users (M) }\end{array}$ & $\begin{array}{l}\text { Delete } \\
\text { Message } \\
\text { Locally }\end{array}$ & $\begin{array}{l}\text { Delete } \\
\text { Message } \\
\text { Globally }\end{array}$ & $\begin{array}{l}\text { Separate } \\
\text { Deletion } \\
\text { Functions }\end{array}$ & Residuals & $\begin{array}{l}\text { Delete } \\
\text { Chat }\end{array}$ & $\begin{array}{l}\text { Ephemeral } \\
\text { Messages }\end{array}$ \\
\hline $\begin{array}{r}\text { Facebook Messenger } \\
\text { Google Hangouts } \\
\text { GroupMe } \\
\text { Line } \\
\text { Signal } \\
\text { Skype } \\
\text { Snapchat } \\
\text { Telegram } \\
\text { Threema } \\
\text { Viber } \\
\text { WeChat } \\
\text { WhatsApp }\end{array}$ & $\begin{array}{r}1300[9] \\
19.23[10] \\
9.22[10] \\
214[9] \\
0.5[11] \\
300[9] \\
255[9] \\
100[9] \\
3.5[12] \\
260[9] \\
963[9] \\
1300[9]\end{array}$ & $\begin{array}{l}0 \\
0 \\
0 \\
0 \\
0 \\
0 \\
0 \\
0 \\
0 \\
0 \\
0 \\
0\end{array}$ & $\begin{array}{l}0 \\
0 \\
0 \\
0 \\
0 \\
0 \\
0 \\
0 \\
0 \\
0 \\
0 \\
0\end{array}$ & $\begin{array}{l}- \\
- \\
- \\
0 \\
0 \\
0 \\
0 \\
0 \\
- \\
0 \\
0\end{array}$ & $\begin{array}{l}0 \\
- \\
0 \\
0 \\
0 \\
0 \\
0 \\
0 \\
0 \\
0 \\
0 \\
0\end{array}$ & $\begin{array}{l}0 \\
0 \\
0 \\
0 \\
0 \\
0 \\
0 \\
0 \\
0 \\
0 \\
0 \\
0\end{array}$ & $\begin{array}{l}0 \\
0 \\
0 \\
0 \\
0 \\
0 \\
0 \\
0 \\
0 \\
0 \\
0 \\
0\end{array}$ \\
\hline
\end{tabular}

time span of 24 hours to delete messages from the logs of all participants involved in a conversation [16].

We say a messenger provides Separate Deletion Functions if it allows the user to select whether the message should be deleted from all conversation histories or only from the sender's history. This mechanism only applies to messengers supporting both types of deleting messages, i.e., locally and globally. If an application supports global deletion and does not provide separate functions, this means that the message can only be removed from all conversation logs at the same time.

\section{B. Additional Properties}

Upon deletion, several messengers display a message or leave another hint that a message was deleted. If a user can identify when messages in a conversation have been deleted, we say the messenger leaves Residuals. For example, WhatsApp displays "This message was deleted" notifications in the recipients' message histories, and "You deleted this message" on the sender's side.

The functionality to delete an entire conversation, referred to as Delete Chat, is an additional feature supported by all mobile instant messaging applications we considered. As mentioned before, in Google Hangouts, this is the only way to delete messages locally.

Some messengers also support the concept of Ephemeral Messages, which are automatically deleted from all message histories after a specific time span. This functionality is not only found in explicitly privacy-oriented applications such as Signal or Telegram, but also in the popular Snapchat app.

\section{Research Questions}

The different messaging applications comprise a variety of implementations of deletion functionalities. We consider this a broad selection of offers made by the application developers to their users. From the opposite perspective, this directly raises the question which features users actually demand for their everyday conversations. Therefore, following our first research question (RQ1), we study how commonly deleting is applied by users, whether there is a need for this functionality, and in particular, which options users prefer, inspired by the currently available options.

In our second research question (RQ2), we examine whether users can correctly assess the capabilities of deletion functions and whether we can identify differences in distinct implementations of these functions. We expect that the variety of implementations of deletion mechanisms is confusing for users. For example, deleting a message in Skype removes messages from all participants' conversations, whereas the identically named function in, e. g., Facebook Messenger only removes messages from the sender's log. Line Messenger is more transparent by providing a prompt stating that the message is only deleted locally, and requiring the user to confirm before the message is eventually deleted. It is unclear how an average user who has not explicitly explored the actual impact of deleting in a particular app can objectively assess what happens when a message is deleted.

\section{METhODOLOGY}

In this section, we discuss the design and methodology of our study in detail. We conducted a between-subject study comprising three test conditions (study groups). During the study, the participants in each condition interact with one particular mobile instant messenger and answer 16 questions on a laptop.

\section{A. Test Conditions}

For our practical study, we assigned participants one of three test conditions based on the different instant messaging applications:

- Skype (version 8.13) deletes messages from the message logs of all participants in the conversation;

- Facebook Messenger (version 151.0) allows the sender to delete messages from their own conversation history only; 
- WhatsApp (version 2.18) allows users to select whether to delete the message just from the sender's conversation history or for all parties involved.

These messengers were selected because they have a large user base and they implement different behaviors of the message deletion functionality, thus representing all sound variations of deleting messages (Table I). All versions correspond to the most current versions available as of February 2018.

\section{B. Study Design}

Our study comprises five steps: (1) Introduction, (2) Practical Task: Writing and Deleting a Message, (3) Questionnaire Part I: Expectations of Deleting Messages, (4) Questionnaire Part II: Reconsidering Effects of Deleting, and (5) Debriefing.

Following the introduction, participants complete the initial practical task of sending and deleting a message using an instant messaging application. Subsequently, participants answer a two-part questionnaire, briefly interrupted by the study supervisors presenting the result of the experiment. We explain these steps in more detail in the following.

Step 1-Introduction: In the first step, we explain to each participant the reason for and purpose of the study as well as the task they will be asked to do. Furthermore, we inform them that no personal data will be collected, how long their participation will approximately take, and what the reward for their participation will be.

Step 2-Practical Task: Writing and Deleting a Message: Next, the first stage of the practical task follows. We ask the participants to write, send, and delete a message using a specific instant messaging service.

For this task, the participants are provided with a mobile phone (Samsung Galaxy S6 running Android 7) with the specific messaging service already opened to keep the task of sending and deleting a message as simple as possible-we do not ask the participants to use their own mobile devices. We use our lab mobile phone in order to create a more controlled environment where the messaging service is installed and working and the contact phone number is already in the contact list. Participants are asked to type an arbitrary message, but if they struggle to come up with a message of their own, we suggest they simply send "hello".

On a second phone, we then show the participants that the message has arrived at the recipient's device, and ask them to delete the message on the device they have sent it from. If necessary, we assist the participants to figure out how to delete the message. At this point, we do not show them the effect of deleting the message on the recipient's device-this will be revealed in Step 4.

Step 3-Questionnaire Part I: Expectations of Deleting Messages: At this point, the participants proceed with a questionnaire on a laptop. It starts with a few warm-up questions about the participants' usage of mobile devices and instant messaging, and whether they delete instant messages and why. We further ask questions about the participants' expectations concerning the experiment-whether the message was deleted everywhere or only from the sending device. Additionally, we ask the participants which deletion behavior they would prefer. Demographic data is also collected in this part of the questionnaire.

The full set of questions and their answers can be found in the Appendix.

Step 4-Questionnaire Part II: Reconsidering Effects of Deleting: In the second part of the practical evaluation, we reveal the outcome of the initial experiment, i.e., we present the message history of the recipient's device to the participants. This allows them to see the effect of deleting the message on the recipient's side.

Subsequently, the participants continue with the second part of the questionnaire, specifically focusing on questions about the message deletion and whether it acted as expected. In the last two questions, we ask the participants whether there should be limitations for deleting messages from the recipient's message history.

These questions are primarily addressed at participants of the WhatsApp and Skype conditions since these messengers allow deleting messages from the recipient's message history.

Step 5-Debriefing: After these final questions, we thank the participants for their participation. If they have any questions about the study, we answer them in this step.

Finally, we delete the entire message history to preserve privacy and to enable the next participant to start with an empty message history.

\section{Pilot Study}

In December 2017, we conducted a pilot study to evaluate the procedure of the study, determine the duration per participant, and test the comprehensibility of the questions. We tested the study on 8 colleagues from a co-located department ( $75 \%$ male, $25 \%$ female, age ranging from 25 to 59 years). The participants did not have any prior knowledge of the study and its goals. As a result, three questions were removed from the questionnaire as they turned out to be somewhat redundant or too imprecise. We also decided to let participants fill out the questionnaire on laptops instead of structured interviews or paper-based questionnaires to avoid errors during data collection and simplify administering the responses.

\section{Study Protocol, Recruitment, and Demographics}

Over a period of three days in February 2018, we collected a total of 135 responses from visitors to the main cafeteria on our university's campus. The main cafeteria is centrally located and frequented by students and staff from all departments. We set up two tables in relatively quiet corners near the two main entrance doors and recruited participants from the passing students and staff. This setup allowed for rather quick recruitment of participants but may also have biased the sample. However, as the cafeteria serves all departments, we expected participants with a wide variety of backgrounds.

Study participants could choose if they preferred to do the study in English or German. $93 \%$ of the participants chose to answer in German. Completing the study took on average five minutes, and we compensated each participant with two chocolate bars regardless of whether they completed the study or aborted early. 
TABLE II. PARTICIPANT DEMOGRAPHICS

\begin{tabular}{|c|c|c|c|c|c|}
\hline & & WhatsApp & Skype & $\begin{array}{l}\text { Facebook } \\
\text { Messenger }\end{array}$ & All Conditions \\
\hline \multirow{4}{*}{ 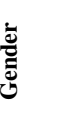 } & Female & 17 & 12 & 11 & $40(32.0 \%)$ \\
\hline & Male & 21 & 28 & 31 & $80(64.0 \%)$ \\
\hline & Other & 0 & 1 & 2 & $3(2.4 \%)$ \\
\hline & No answer & 1 & 0 & 1 & $2(1.6 \%)$ \\
\hline \multirow{5}{*}{ 适 } & $<\mathbf{2 0}$ & 6 & 10 & 9 & $25(20.0 \%)$ \\
\hline & 20-34 & 32 & 26 & 30 & $88(70.4 \%)$ \\
\hline & $35-49$ & 1 & 3 & 4 & $8(6.4 \%)$ \\
\hline & $\geq \mathbf{5 0}$ & 0 & 2 & 1 & $3(2.4 \%)$ \\
\hline & No answer & 0 & 0 & 1 & $1(0.8 \%)$ \\
\hline \multirow{6}{*}{ 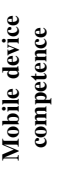 } & (beginner) 1 & 0 & 1 & 0 & $1(0.8 \%)$ \\
\hline & 2 & 3 & 3 & 5 & $11(8.8 \%)$ \\
\hline & 3 & 7 & 11 & 12 & $30(24.0 \%)$ \\
\hline & 4 & 16 & 15 & 19 & $50(40.0 \%)$ \\
\hline & (expert) 5 & 11 & 10 & 7 & $28(22.4 \%)$ \\
\hline & No answer & 2 & 1 & 2 & $5(4.0 \%)$ \\
\hline \multicolumn{2}{|c|}{ Total \# Participants } & 39 & 41 & 45 & $125(100.0 \%)$ \\
\hline
\end{tabular}

Although all participants completed the study, we discarded 10 responses because of incomplete answers, resulting in 125 responses we used in the evaluation. The participants were randomly assigned one of the three conditions.

When the participants answered the questionnaire, the supervisors kept their distance in order to not create additional pressure, while staying available for questions. We did not count the actual number of questions raised by participants, but we estimate that less than 5 participants asked for clarification on survey questions.

We collected demographic data from the study participants. $32 \%$ of the participants stated to be female and $64 \%$ identified as male. The median age is 25 years in a range from 18 to 75 years. Table II summarizes the response to the demographic questions.

We also asked the participants to self-estimate their proficiency in using mobile devices on a five-point scale from beginner (1) to expert (5). According to their answers, more than $60 \%$ of the participants rated their experience in using mobile devices as 4 or 5 .

\section{E. Ethical Considerations}

Our university does not have an IRB or ethics board which covers the type of our study. However, we have taken great care to adhere to principles of ethical research. Our study was designed such that it did not contain deceiving questions. In case participants asked immediately after deleting the message how the deletion affected the recipient's message history, we asked them to be patient until they had completed the first part of the questionnaire. Furthermore, we did not store any data which would allow us to link participants to their responses.

In the recruitment process, each participant was informed that they were participating in a scientific study, about the purpose of the study, the possibility to withdraw at any time without giving any reasons, and that no personally identifying information would be stored.

At the beginning of the questionnaire, the participants were shown an introductory text summarizing the information previously given orally during recruitment.

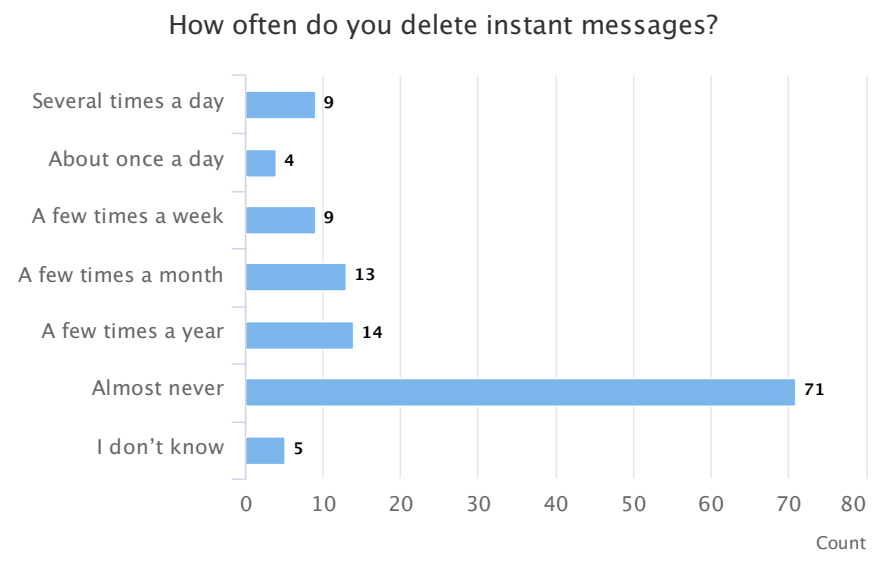

Fig. 2. Answers to $Q 4$ (How often do you delete instant messages?)

We also informed the participants about the estimated duration of the study (approx. five minutes) and their compensation (two chocolate bars). However, some participants required up to 10 minutes or more because they provided detailed answers to the free text questions. Answering these questions was not mandatory and could be omitted. The demographic questions were also completely optional, and the participants could skip them without providing an answer or choose the I prefer not to answer option.

\section{RESULTS}

In this section, we present the results of our study and discuss the results. We report the findings concerning the participants' expectations of deleting messages, based on the questionnaire. The results from the practical task to delete a message are presented and analyzed if and to what extent users correctly assess the actual capabilities of deletion functionality.

\section{A. User Expectations of Message Deletion}

First, we consider the participants' expectations of deleting messages as expressed in the questionnaire, in particular in Questions $Q 4$ to $Q 7, Q 15$, and $Q 16$. Here we are faced with subjective wishes and concerns of users. We are interested in whether users actually use message deletion features in their daily lives, how often they use them, with what intentions, and what technical implementation they think best fits their needs.

Frequency of Message Deletion: To learn about the usage of message deletion, we directly asked the participants how often they use the deletion functions in instant messaging ( $Q 4$ : How often do you delete instant messages?). Possible answers ranged from "Several times a day" to "Almost never" and included "I don't know".

The distribution of answers is shown in Figure 2. On the one hand, the results show that, on average, message deletion is a relatively infrequent event: $56.8 \%$ of users $(k=71)$ almost never delete messages, and only $10.4 \%(k=13)$ of participants use it on a daily basis.

However, the answers also show that $39.2 \%(k=49)$ of participants have used the feature of message deletion. These numbers also indicate that the usage patterns are widely 
What are your reasons for deleting messages?

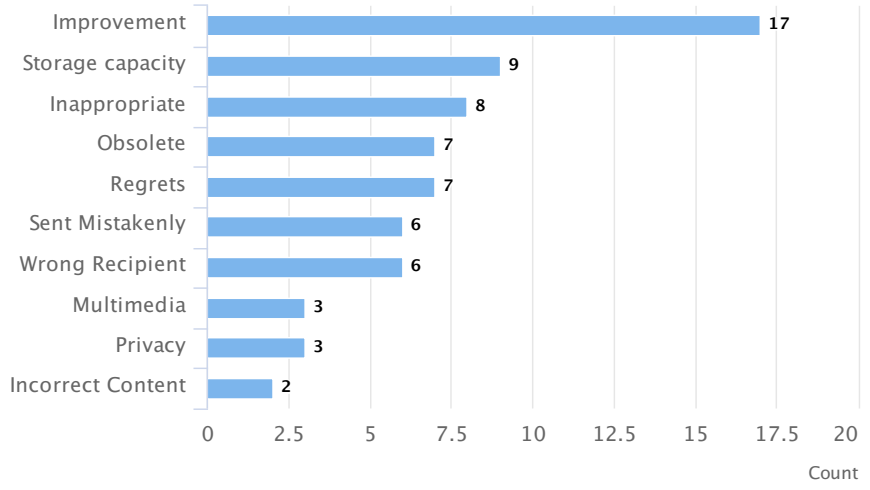

Fig. 3. Answers to $Q 5$ (What are your reasons for deleting messages?). We collected a total of 42 responses to which we assigned 68 tags.

different: We find about equal numbers of participants using message deletion "a few times a year/month/week" as well as "several times a day" (each approx. 10\%). Thus, we expect diverse answers for the reasons to delete.

Reasons for Message Deletion: We used a free text field to let participants describe their reasons for deleting messages, as we expected a wide variety of answers. We used a grounded coding approach, where three researchers independently derived codes from the answers. We compared and merged the codes, which resulted in 11 different final codes, and applied these to the answers. One statement could be tagged with multiple codes, and we finally obtained 68 tags to 42 different answers. Three answers were left out as the coders agreed that they were too ambiguous. The resulting category tags are displayed in Figure 3.

The most frequent reasons for message deletion were improvements $(k=17)$ of the message, such as grammar or spelling corrections but also wording improvements, and issues with storage capacity $(k=9)$-three responses explicitly referred to multimedia content such as images or videos. Inappropriate messages, e.g., messages that have been sent thoughtlessly and could offend the recipient, were named by eight participants. The latter is also related to senders who regret that they had sent a particular message $(k=7)$. Six participants further explained that they deleted messages because they had sent them to a wrong recipient. However, explicit privacy reasons, such as unwanted further access to the message contents, were only mentioned three times.

In summary, we can distinguish three principal categories: Users delete messages mainly for linguistic, storage-related, or privacy-enhancing reasons. Other reasons for deleting messages we also consider relevant for user privacy include messages being inappropriate, obsolete, regretted by the sender, sent mistakenly, to the wrong recipient, or containing incorrect content. Thus, $57.4 \%(k=39)$ of the tags are somehow privacy-related, distributed across $61.9 \%(k=26)$ of the $(n=42)$ useful free text answers we have received, that is $20.8 \%$ of all 125 participants.

Favored Option for Deleting Messages: We have asked users about their favored variant for deleting messages $(Q 7$ :
TABLE III. ANSWERS TO $Q 7$ (WHICH OF THE FOLLOWING DO YOU PREFER WHEN YOU DELETE A MESSAGE?)

\begin{tabular}{lr}
\hline Preferred Option & Count \\
\hline The message is deleted from my device only & $12(9.6 \%)$ \\
The message is deleted from recipient's device only & $8(6.4 \%)$ \\
The message is deleted from both devices & $54(43.2 \%)$ \\
For each message, I can choose where to delete from & $51(40.8 \%)$ \\
\hline
\end{tabular}

Which of the following do you prefer when you delete a message?), i.e., from which message histories they prefer messages to be removed. Participants could pick one of four predefined answers. The results are listed in Table III. The majority of participants $(84 \%, k=105)$ preferred either the message to be deleted from both the sender's and recipient's logs or to be given the choice between global and local deletion whenever they delete a message. These numbers are supported by our observations of the study participants who were assigned to the WhatsApp condition in the experiment. 36 of them chose the Delete for Everyone option, while only three decided to remove the message from their message history only. This indicates two things: First, our results suggest that the majority of users who have decided to delete a message expect deletion to have global effects. Second, there also appears to be a need for a selection mechanism on a permessage-basis, which implies that users desire more granular functionality and also higher transparency when they delete messages.

User Expectation of the Limitations of Deleting Messages: We have asked users whether the (global) delete functionality in instant messaging should be limited (Q15: Do you think the delete function should be limited?). We suggested examples such as time limitation, message order, or message status (read vs. unread). While 39 participants $(31.2 \%)$ agreed with this, we received 86 negative answers. The 39 participants who supported such a limitation were asked to further specify the type of limitation (Q16: How should the delete function be limited?). We coded their free text answers into six different categories (again, following a grounded approach with three coders). One answer could be labeled with multiple codes. While we did not categorize seven answers as we agreed that these were too ambiguous or not related to the question, we assigned a total of 35 tags to 32 different answers. The distribution of the answers is illustrated in Figure 4.

The majority of participants proposed to either allow deletion only for unread messages $(k=12)$ or to limit the deletion functionality based on time $(k=11)$. Five participants opposed message deletion in general, and four participants proposed to restrict deletion to the latest message only. This restriction is actually implemented in the WeChat messenger (which we did not cover in our study) and appears interesting in that it keeps the conversation history consistent. Deleting a message after one or more follow-up messages can change the entire context of the subsequent conversation. Quite interestingly, two participants suggested that for each conversation all partners should be required to consent whether and under which circumstances messages can be removed from the conversation history. 
How should the delete function be limited?

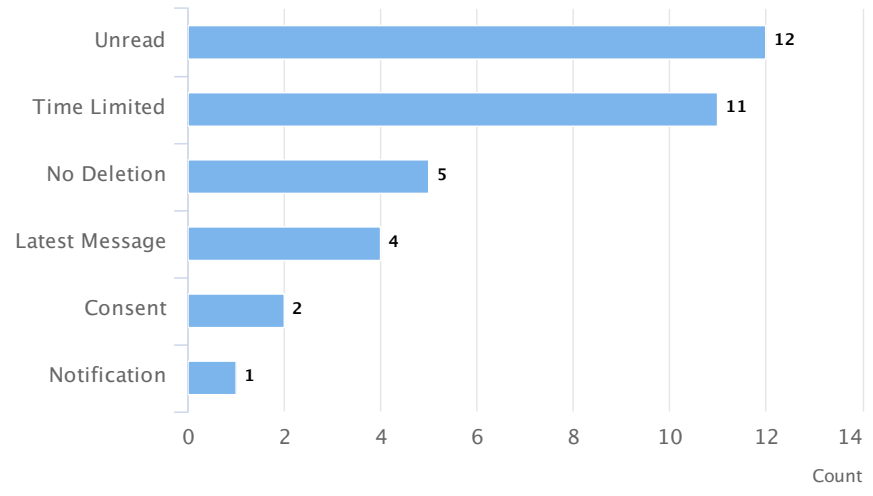

Fig. 4. Answers to $Q 16$ (How should the deletion function be limited?). We collected a total of 32 responses to which we assigned 35 tags.

TABLE IV. ANSWERS TO Q13 (DOES THIS RESULT MATCH YOUR EXPECTATIONS?), SEPARATED BY MESSENGER APPLICATIONS

\begin{tabular}{lccr}
\hline Messenger & participants & yes & no \\
\hline Facebook & 45 & $32(71.1 \%)$ & $13(28.9 \%)$ \\
Skype & 41 & $20(48.8 \%)$ & $21(51.2 \%)$ \\
WhatsApp & 39 & $31(79.5 \%)$ & $8(20.5 \%)$ \\
\hline Total & 125 & $83(66.4 \%)$ & $42(33.6 \%)$ \\
\hline
\end{tabular}

\section{B. Expectation Matching in Real Implementations}

We also investigate how well the messenger interfaces communicate the type of deletion they implement, specifically whether messages are deleted on the sender's device only, the receivers' device only, or both. In Step 4 of the study, we disclosed to the participants whether the message they had sent and deleted was still available on the recipient's device. We then asked the participants if the result matched their expectations (Q13: Does this result match your expectations?). Additionally, the participants could provide a free text answer to specify differences between their expectations and the result.

The results are summarized in Table IV. Overall, $66.4 \%$ of the participants $(k=83)$ stated that the observed behavior matched their expectation; however, the results depend on which messenger was used. For Facebook Messenger $71 \%$ agreed, for Skype $49 \%$ agreed, and for WhatsApp $80 \%$ agreed.

We used a chi-square test of independence to test if these differences among the three messengers are statistically significant and found a significant influence $\left(\chi^{2}=9.1468\right.$, $d f=2, p=0.01032$ ). For post-hoc testing we used chisquare tests on pairs of messengers and applied corrections for multiple testing. We used Bonferroni correction as a conservative choice, as the number of tests is small. Among the post-hoc tests on pairs of messengers, we found significant differences between Skype and WhatsApp $\left(\chi^{2}=6.8806\right.$, $d f=1, p=0.02613)$. Participants in the WhatsApp condition could better assess the effects of message deletion by $30 \%$ than participants in the Skype condition. A summary of the test results is shown in Table V. We used a significance level of $\alpha=0.05$.
Why does this result not match your expectations?

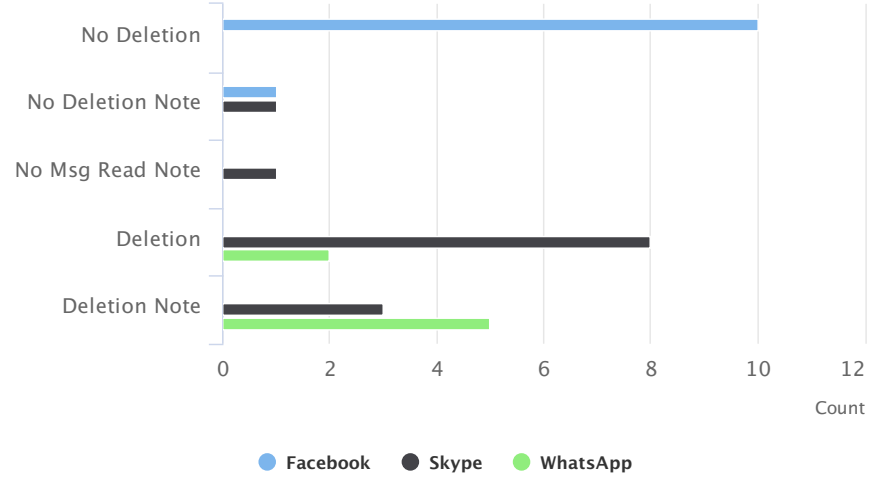

Fig. 5. Reasons for diverging expectations, grouped by the three messenger applications used in the study. We collected a total of 31 responses.

TABLE V. RESULTS OF TESTS OF INDEPENDENCE OF MESSENGER AND EXPECTATION MATCHING.

\begin{tabular}{lccc}
\hline Messenger Combination & $\chi^{2}$ & $d f$ & $p$ \\
\hline Facebook vs. Skype & 3.58980 & 1 & 0.17439 \\
Facebook vs. WhatsApp & 0.39886 & 1 & 1.00000 \\
Skype vs. WhatsApp & 6.88060 & 1 & 0.02613 \\
\hline Omnibus (All Messengers) & 9.14680 & 2 & 0.01032 \\
\hline
\end{tabular}

Reasons for Non-matching Expectations: Prior to the experiment, we expected a higher rate of expectation matching, particularly in the WhatsApp condition, whose members were able to explicitly choose which message history they would like to delete the message from. Therefore, we analyze the reasons why the expectations did not match. Participants could specify in detail the reasons why and how the result differed from what they had expected (Q14: Why does this result match your expectations? Why not?).

We received 32 free text answers and coded them, again following a grounded approach with three coders. One participant noted to have expected a prompt to choose whether the message should be deleted locally or globally. The coders agreed to drop this answer as it is not related to the disclosure of the result at the end of the experiment. We categorized the remaining 31 answers into five disjoint categories as illustrated in Figure 5.

The majority of responses $(k=20)$ simply referred to surprises because a message was deleted (Deletion) or because it was not deleted (No Deletion). Another 10 participants' answers referred to the delete notification as the reason why the outcome did not match their expectations. The categories do not apply to all three messengers equally, e.g., only participants who used Facebook Messenger could expect a deletion that did not occur $(k=10)$. Quite interestingly, the answers indicate that the expectation mismatch partially originated from the notification that a message has been deleted (Skype: 3, WhatsApp: 5).

\section{Limitations}

We have planned and conducted our study thoroughly. However, our sampling approach introduces certain limitations. 
We have reached a large number of participants with moderate effort, but this resulted in a sample biased towards younger people who have (at their own judgment) higher than average experience with mobile devices. For better general applicability of our results, a sample with a more representative age distribution and more objective assessment of experience would be desirable.

The study environment was rather busy compared to an inlab setup, which is, however, more representative for normal smartphone usage.

In our survey, several questions only offered binary (yes / no) answer options. Most of the binary answers were used in the warm-up questions. Only the answers to Q13 were used for quantitative evaluation, and these are supported by the qualitative answers to Q14. Answer ranges based, e.g., on Likert scales might have been a better instrument to capture varying levels of people's opinions. Our goal was to obtain a coarse estimation of expectations on message deletion, not necessarily representing all possible aspects. The use of a survey with predominantly closed questions facilitated the analysis compared to interviews, at the expense of limiting the participants' ability to express differentiated answers.

The test conditions were also limiting the applicability of our findings, in that we only tested three different implementations of messengers and did not cover all deletion features such as ephemeral messages. The three messengers we tested are, however, among the most popular ones and comprise different realizations of the deletion functionality.

\section{Discussion}

The term "deleting messages" can be ambiguous as it can be unclear whether messages are removed from the sender's or the recipient's log, or both. Our results have shown that improper design of the interface can lead to confusion. Participants in our study could not correctly assess the actual effects of deleting a message in an application that does not adequately explain its functionality.

WhatsApp's implementation, comprising an explicit choice, is more transparent than implementations in other applications, in that users can directly decide whether they prefer a message to be deleted only locally or also on the recipient's side. While WhatsApp's implementation is the most transparent one, it also meets the desire expressed by a significant number of participants to be able to explicitly choose between global and local deletion-it can be considered a best practice.

It is still to be investigated whether a more clear description of the delete function on the user interface can better clarify where messages are deleted, even when no choice is given to the user. One example could be the Line messenger, which explicitly advises a user that the respective message is only deleted from the user's local conversation history and that the recipients will still be able to read it.

It is interesting that a majority of participants $(68.8 \%)$ did not express an explicit desire for limits on the delete functionality. There are good reasons for such limits, as preserving a consistent conversation is desirable. The limitation to seven minutes originally implemented by WhatsApp appears appropriate according to the majority of reasons users stated for deleting messages. This time span is sufficient to correct or improve messages and to withdraw messages that have been sent mistakenly or to a wrong recipient. However, it remains unclear how this limitation was determined. In early March 2018, the time limit for message deletion in WhatsApp was extended to 68 minutes and 16 seconds (i. e., $2^{12}$ seconds) [13], which suggests that the rationale for the concrete time limit may also be purely technical.

Another interesting proposal-yet not implemented in any of the messenger applications we have examined-might be consent-based deleting. In such a scenario, messages can only be deleted if all participants in the conversation have explicitly stated so beforehand, on a per-conversation basis. Such a mechanism could balance individual interests of both the sender (to keep control of potentially sensitive data) and the receiver (to keep track of the conversation). Unlimited availability of the functionality to delete messages could evoke malicious deletion, e.g., to alter the context of a conversation retroactively. Consent-based deletion might help to reduce these threats.

These examples show how the user experience of messaging applications could be improved, in particular, concerning message deletion. Application developers could provide a notification where a message has been deleted from, or implement a dialog for explicit selection, to improve users' understanding of the capabilities of deletion functionality.

\section{A. Future Work}

In the future, the study could be repeated with a larger and more heterogeneous sample with different age ranges and educational backgrounds to review our findings for generalizability beyond a university context. A study replication could also cover additional messengers to capture a wider range of deletion features, e.g., ephemeral messages.

While we considered aspects such as reasons for deletion, frequency, etc. only independent of each other, future research could explore dependencies, i. e., whether people who delete messages more frequently have different reasons for it.

While initially gathering information about the variety of deletion features, we observed that there are diverse names for these functions across different messengers (e. g., Delete, Recall, Unsend). This raises the question whether user perception differs depending on how the functionality is named.

\section{RELATED WORK}

We have explored whether users understand how message deletion works in instant messengers. Apart from users' perceptions, also questions about the security of the implementations of such features arise. Messenger security, for example, with focus on their end-to-end encryption, has been well-studied and shown to have flaws [17]-[19]. A broad overview of security features in instant messaging is provided by Unger et al. [20].

For several years, the privacy paradox has found remarkable attention-users' attitude concerning their online privacy differs from how they actually behave in online contexts [21][23]. One explanation for this phenomenon might be that 
privacy is just considered a feature that can be traded in for other valuable goods or services [24]-[26].

Other findings suggest that users cannot review the entire consequences of their behavior because the systems they use do not adequately inform them. Abu-Salma et al. [27] examined the use of various security features in Telegram, along with a usability inspection, and revealed that a sparse presentation of multiple security alternatives could lead to confusion among users. In a cloud computing context, Ramokapane et al. [28] have found that users fail to delete contents because of poorly designed interfaces. Acquisti et al. [29] provide an overview of how users can be better assisted in their security choices.

Independent from shortcomings in implementations of user interfaces, users are however able to differentiate in their use of online communication. Sleeper et al. [30] found how users select different messaging or communication channels depending on the purpose or target audience. Ruoti et al. [31] showed that users reflect their online posture in the light of never being perfectly safe on the Internet.

Our findings on reasons for message deletion are similar to the results of Almuhimedi et al. [32] who conducted a largescale study on deleted tweets. We have found that regrets and contents being considered inappropriate were among the major reasons for users to delete instant messages. Several studies have already considered users' regrets about their postings in Online Social Networks and examined reasons, consequences, and coping strategies [33]-[35], but without mainly focusing on instant messaging. Reasons for content deletion or dereferencing have also been explored in the context of social networks [36], [37]. It has turned out that dissociation and hiding can be appropriate strategies as alternatives to deleting in social networks, where users have only little control over the dissemination of their contents once they have been released [38].

\section{CONCLUSION}

In this work, we studied users' expectations towards the deletion functionality in instant messengers. Specifically, we investigated whether users could accurately determine from which conversation histories their messages were removed upon performing a deletion. We tested three different messengers (WhatsApp, Skype, Facebook Messenger) in a user study with 125 participants.

Deletion functionality in WhatsApp is different from the other two messengers in that users can explicitly select whether they want to delete a message on their local conversation history or also from the recipients' logs. We found that this led to a $30 \%$ higher rate of correctly predicting the effects of deleting messages. We suggest that developers of other instant messaging applications describe the effect of message deletion more explicitly, e.g., by providing a dialog for selection as in WhatsApp, or include a notification indicating where the message has been deleted from.

\section{ACKNOWLEDGMENT}

The authors would like to thank Henry Hosseini and Eduard Leonhardt for their help with conducting the study.
This research was supported in parts by the BMBF InStruct project under grant 16KIS0581 and the MKW-NRW Research Training Group SecHuman.

\section{REFERENCES}

[1] Statista GmbH, "Number of Mobile Phone Messaging App Users Worldwide From 2016 to 2021 (in Billions)," Jul. 2017, https://www. statista.com/statistics / 483255/number- of - mobile - messaging - users worldwide/, as of April 9, 2018.

[2] Elle Hunt, "I Sent A Compromising Message to the Wrong Person. How Will I Ever Recover? ," Apr. 2017, https://www.theguardian.com/ culture/2017/apr/28/i- sent- a-compromising- message- to- the- wrongperson-how-will-i-ever-recover.

[3] Alice Johnston, "Now That's Awkward! Hilarious Texts Show What Happens When You Send a Message to the WRONG Person," Oct. 2016, http://www. dailymail.co.uk/femail/article-3871302/Hilarioustexts-happens-send-message-WRONG-person.html.

[4] European Parliament, "Regulation (EU) 2016/679 of the European Parliament and of the Council of 27 April 2016 on the Protection of Natural Persons With Regard to the Processing of Personal Data and the Free Movement of Such Data, and Repealing Directive 95/46/EC (General Data Protection Regulation)," Official Journal of the European Union, vol. 59, 2016.

[5] European Union, "Factsheet on the "Right to be Forgotten" Ruling (C131/12)," May 2014.

[6] Aatif Sulleyman, "WhatsApp's 'Delete for Everyone' Feature Lets You Unsend Embarrassing Messages: Here's How to Use it," Oct. 2017, https://www.independent.co.uk/life-style/gadgets-and-tech/news/ whatsapp- delete- unsend- message-for- everyone- before-read-how-touse-how-does-it-work-a8022816.html.

[7] Emily Price, "Now WhatsApp Allows You to Delete Messages," Oct. 2017, http://fortune.com/2017/10/27/whatsapp-delete-messages/.

[8] A. Whitten and J. D. Tygar, "Why Johnny Can't Encrypt: A Usability Evaluation of PGP 5.0," in Proceedings of the 8th Conference on USENIX Security Symposium - Volume 8. Berkeley, CA, USA: USENIX Association, 1999, pp. 14-14.

[9] Statista GmbH, "Most Famous Social Network Sites Worldwide as of September 2017, Ranked by Number of Active Users (in Millions)," Sep. 2017, https://www.statista.com/statistics/272014/global-socialnetworks-ranked-by-number-of-users/, as of April 9, 2018.

[10] _ "Most Popular Mobile Messaging Apps in the United States as of July 2017, by Monthly Active Users (in Millions)," Jul. 2017, https:// www.statista.com/statistics/350461/mobile-messenger-app-usage-usa/, as of April 9, 2018.

[11] Jeff John Roberts, "Here Are the Most Popular Apps for Secure Messages," Jan. 2017, https://fortune.com/2017/01/17/most-popularsecure-apps/, as of April 9, 2018.

[12] Rob Price, "Germany's Most Popular Paid App is a Secure Messenger Loved by Millions - Now it's Taking on the US," Jun. 2015, http: //uk.businessinsider.com/threema-encryption-messaging-app-americalaunch-isis-2015-6, as of April 9, 2018.

[13] Jason Murdock, "WhatsApp New Feature: Users May Soon Have an Hour to Recall Embarrassing Chats," Mar. 2018, http://www.newsweek. com/whatsapp-users-may-soon-have-hour-delete-embarrassing-chatsgroups-830675.

[14] Amanda Connolly, "WhatsApp Turns on Unsend Feature to Let You Delete Embarrassing Messages," Nov. 2015, https://thenextweb.com/ apps/2015/11/26/finally-2/.

[15] Kaylene Hong, "WeChat Gets the Option to Recall Sent Messages, and One-Click Payment Transfer in China," Jun. 2014, https: // thenextweb.com/apps/2014/06/23/wechat - gets - inspired - by - the ephemeral-messaging-trend-now-lets-you-recall-sent-messages/.

[16] Natasha Lomas, "Line Adds Unsend For Recalling Missent Messages," Dec. 2017, https://beta.techcrunch.com/2017/12/13/line-adds-unsendfor-recalling-missent-messages/.

[17] A. Herzberg and H. Leibowitz, "Can Johnny Finally Encrypt?: Evaluating E2E-encryption in Popular IM Applications," in Proceedings of the 6th Workshop on Socio-Technical Aspects in Security and Trust, ser. STAST '16. New York, NY, USA: ACM, 2016, pp. 17-28. 
[18] P. Rösler, C. Mainka, and J. Schwenk, "More is Less: On the Endto-End Security of Group Chats in Signal, WhatsApp, and Threema," in 2018 IEEE European Symposium on Security and Privacy. IEEE Computer Society, April 2018.

[19] T. Frosch, C. Mainka, C. Bader, F. Bergsma, J. Schwenk, and T. Holz, "How Secure is TextSecure?" in 2016 IEEE European Symposium on Security and Privacy (EuroS\&P). IEEE Computer Society, March 2016, pp. 457-472.

[20] N. Unger, S. Dechand, J. Bonneau, S. Fahl, H. Perl, I. Goldberg, and M. Smith, "SoK: Secure Messaging," in 2015 IEEE Symposium on Security and Privacy. IEEE Computer Society, May 2015, pp. 232 249.

[21] S. B. Barnes, "A Privacy Paradox: Social Networking in the United States," First Monday, vol. 11, no. 9, 2006, available online https:// journals.uic.edu/ojs/index.php/fm/article/view/1394/1312, as of April 9, 2018

[22] S. Utz and N. C. Krämer, "The Privacy Paradox on Social Network Sites Revisited: The Role of Individual Characteristics and Group Norms," Cyberpsychology: Journal of Psychosocial Research on Cyberspace, vol. 3, no. 2, 2009.

[23] S. Kokolakis, "Privacy Attitudes and Privacy Behaviour: A Review of Current Research on the Privacy Paradox Phenomenon," Computers \& Security, vol. 64, pp. 122-134, 2017.

[24] J. Y. Tsai, S. Egelman, L. Cranor, and A. Acquisti, "The Effect of Online Privacy Information on Purchasing Behavior: An Experimental Study," Information Systems Research, vol. 22, no. 2, pp. 254-268, Jun. 2011

[25] S. Athey, C. Catalini, and C. Tucker, "The Digital Privacy Paradox: Small Money, Small Costs, Small Talk," National Bureau of Economic Research, Tech. Rep., 2017.

[26] Y. Pu and J. Grossklags, "Valuating Friends' Privacy: Does Anonymity of Sharing Personal Data Matter?" in Thirteenth Symposium on Usable Privacy and Security (SOUPS 2017). Santa Clara, CA: USENIX Association, 2017, pp. 339-355.

[27] R. Abu-Salma, K. Krol, S. E. Parkin, V. Koh, K. K. Kwan, J. Mahboob, Z. Traboulsi, and M. A. Sasse, "The Security Blanket of the Chat World: An Analytic Evaluation and a User Study of Telegram," in Second European Workshop on Usable Security (EuroUSEC 2017). Paris, France: IEEE, 2017.

[28] K. M. Ramokapane, A. Rashid, and J. M. Such, “"' Feel Stupid I Can't Delete...": A Study of Users' Cloud Deletion Practices and Coping Strategies," in Thirteenth Symposium on Usable Privacy and Security (SOUPS 2017). Santa Clara, CA: USENIX Association, 2017, pp. 241-256.
[29] A. Acquisti, I. Adjerid, R. Balebako, L. Brandimarte, L. F. Cranor, S. Komanduri, P. G. Leon, N. Sadeh, F. Schaub, M. Sleeper, Y. Wang, and S. Wilson, "Nudges for Privacy and Security: Understanding and Assisting Users' Choices Online," ACM Comput. Surv., vol. 50, no. 3, pp. 44:1-44:41, Aug. 2017.

[30] M. Sleeper, W. Melicher, H. Habib, L. Bauer, L. F. Cranor, and M. L. Mazurek, "Sharing Personal Content Online: Exploring Channel Choice and Multi-Channel Behaviors," in CHI Conference on Human Factor in Computing Systems, ser. CHI '16. Santa Clara, California, USA: ACM, May 2016, pp. 101-112.

[31] S. Ruoti, T. Monson, J. Wu, D. Zappala, and K. Seamons, "Weighing Context and Trade-offs: How Suburban Adults Selected Their Online Security Posture," in Thirteenth Symposium on Usable Privacy and Security (SOUPS 2017). Santa Clara, CA: USENIX Association, 2017, pp. 211-228.

[32] H. Almuhimedi, S. Wilson, B. Liu, N. Sadeh, and A. Acquisti, "Tweets Are Forever: A Large-scale Quantitative Analysis of Deleted Tweets," in Proceedings of the 2013 Conference on Computer Supported Cooperative Work, ser. CSCW'13. New York, NY, USA: ACM, 2013, pp. 897-908.

[33] M. Sleeper, J. Cranshaw, P. G. Kelley, B. Ur, A. Acquisti, L. F. Cranor, and N. Sadeh, "'I Read My Twitter the Next Morning and Was Astonished": A Conversational Perspective on Twitter Regrets," in Proceedings of the SIGCHI Conference on Human Factors in Computing Systems, ser. CHI '13. New York, NY, USA: ACM, 2013, pp. 3277-3286.

[34] Y. Wang, G. Norcie, S. Komanduri, A. Acquisti, P. G. Leon, and L. F. Cranor, "'I Regretted the Minute I Pressed Share": A Qualitative Study of Regrets on Facebook," in Proceedings of the Seventh Symposium on Usable Privacy and Security, ser. SOUPS '11. New York, NY, USA: ACM, 2011, pp. 10:1-10:16.

[35] S. Guha, E. P. Baumer, and G. K. Gay, "Regrets, I've Had a Few: When Regretful Experiences Do (and Don'T) Compel Users to Leave Facebook," in Proceedings of the 2018 ACM Conference on Supporting Groupwork, ser. GROUP' 18. New York, NY, USA: ACM, 2018, pp. 166-177.

[36] O. Ayalon and E. Toch, "Retrospective Privacy: Managing Longitudinal Privacy in Online Social Networks," in Symposium on Usable Privacy and Security, ser. SOUPS '13. Newcastle, United Kingdom: ACM, Jul. 2013, pp. 4:1-4:13.

[37] J. Birnholtz, M. Burke, and A. Steele, "Untagging on Social Media: Who Untags, What do They Untag, and Why?" Computers in Human Behavior, vol. 69, pp. 166 - 173, 2017.

[38] M. Bishop, E. R. Butler, K. Butler, C. Gates, and S. Greenspan, "Forgive and Forget: Return to Obscurity," in New Security Paradigms Workshop, ser. NSPW '13. Banff, Alberta, Canada: ACM, Sep. 2013, pp. 1-10. 


\section{A. Questionnaire Instructions}

This study by Ruhr University Bochum's Mobile Security Group investigates how users use and perceive the deleting functionality in instant messengers on mobile devices. We just asked you to write and delete a message in an instant messaging app. This survey will ask you some questions about how you use the "delete message" feature in mobile instant messengers and how you expect this feature to work. If you have any questions about the survey, feel free to ask any time! Privacy Policy: All data we collect in the course of this study is treated confidentially. We store all the answers you have entered for further evaluation and analysis. We also measure the total time it takes you to complete the survey and perform the experimental tasks. All data we have collected is stored anonymously such that it is not possible to connect the data to your person at any point in time. Please note that your choice to participate in this study is completely voluntary. You are free to withdraw from the study at any time, and we will discard all of your data and not analyze or store it. If you agree with this procedure, click the Next button to begin.

\section{B. Questionnaire Page 1}

Q1: Do you frequently use instant messaging (e.g., WhatsApp or Snapchat) on a mobile device (e. g., smart phone or tablet computer)? (frequently means several times a month)

\begin{tabular}{lcccrr}
\hline & WhatsApp & Skype & Facebook Messenger & \multicolumn{2}{c}{ All Conditions } \\
\hline Yes & 38 & 38 & 44 & 120 & $(96.0 \%)$ \\
No & 1 & 3 & 1 & 5 & $(4.0 \%)$ \\
\hline
\end{tabular}

Q2: Which mobile operating systems do you use? (Multiple answers possible)

\begin{tabular}{lcccc}
\hline & WhatsApp & Skype & Facebook Messenger & All Conditions \\
\hline Android & 26 & 17 & 33 & 76 \\
iOS & 12 & 22 & 15 & 49 \\
Windows Phone & 6 & 1 & 1 & 8 \\
Other & 1 & 1 & 1 & 3 \\
\hline
\end{tabular}

Q3: Which instant messaging services do you use? (Multiple answers possible)

\begin{tabular}{lcccc}
\hline & WhatsApp & Skype & Facebook Messenger & All Conditions \\
\hline Facebook Messenger & 20 & 22 & 21 & 63 \\
Google Hangouts & 1 & 3 & 2 & 6 \\
GroupMe & 0 & 0 & 0 & 0 \\
Line & 0 & 1 & 0 & 1 \\
Apple Messages & 9 & 9 & 5 & 3 \\
QQ Mobile & 1 & 1 & 1 & 14 \\
Signal & 4 & 6 & 4 & 36 \\
Skype & 15 & 12 & 9 & 33 \\
Snapchat & 10 & 12 & 11 & 41 \\
Telegram & 11 & 19 & 11 & 8 \\
Threema & 3 & 6 & 4 & 7 \\
Viber & 4 & 3 & 1 & 114 \\
WeChat & 2 & 3 & 2 & 13 \\
WhatsApp & 34 & 39 & 41 & 6 \\
Other & 3 & 4 & 6 & \\
\hline
\end{tabular}




\section{Questionnaire Page 2}

Q4: How often do you delete instant messages?

\begin{tabular}{lccccr}
\hline & WhatsApp & Skype & Facebook Messenger & \multicolumn{2}{c}{ All Conditions } \\
\hline Several times a day & 2 & 3 & 4 & 9 & $(7.2 \%)$ \\
About once a day & 1 & 1 & 2 & 4 & $(3.2 \%)$ \\
A few times a week & 3 & 2 & 4 & 9 & $(7.2 \%)$ \\
A few times a month & 4 & 6 & 3 & 13 & $(10.4 \%)$ \\
A few times a year & 4 & 4 & 6 & 14 & $(11.2 \%)$ \\
Almost never & 23 & 24 & 24 & 71 & $(56.8 \%)$ \\
I don't know & 2 & 1 & 2 & 5 & $(4.0 \%)$ \\
\hline
\end{tabular}

Q5: What are your reasons for deleting messages?

- Free text

\section{Questionnaire Page 3}

Q6: We just asked you to send a message and then to delete it. What do you think - where has the message been deleted?

\begin{tabular}{lcccc}
\hline & WhatsApp & Skype & Facebook Messenger & All Conditions \\
\hline From the sender's device & 32 & 35 & 45 & 112 \\
From the recipient's device & 29 & 12 & 7 & 48 \\
Other & 4 & 4 & 1 & 9 \\
\hline
\end{tabular}

Q7: Which of the following do you prefer when you delete a message?

\begin{tabular}{|c|c|c|c|c|}
\hline & WhatsApp & Skype & $\begin{array}{c}\text { Facebook } \\
\text { Messenger }\end{array}$ & $\begin{array}{c}\text { All } \\
\text { Conditions }\end{array}$ \\
\hline The message is deleted from my device only. & 3 & 3 & 6 & $12 \quad(9.6 \%)$ \\
\hline The message is deleted from recipient's device only. & 2 & 3 & 3 & $8(6.4 \%)$ \\
\hline The message is deleted from both devices. & 16 & 19 & 19 & $54(43.2 \%)$ \\
\hline For each message, I can choose where to delete the message from. & 18 & 16 & 17 & $51(40.8 \%)$ \\
\hline
\end{tabular}

Q8: Do you want to be notified if the recipient has already read the message?

\begin{tabular}{lcccll}
\hline & WhatsApp & Skype & Facebook Messenger & \multicolumn{2}{l}{ All Conditions } \\
\hline Yes & 35 & 31 & 31 & 97 & $(77.6 \%)$ \\
No & 4 & 10 & 14 & 28 & $(22.4 \%)$ \\
\hline
\end{tabular}

Q9: Do you think that the recipient should be told that the message has been deleted (e. g., through a "message deleted" hint)?

\begin{tabular}{lccccr}
\hline & WhatsApp & Skype & Facebook Messenger & All Conditions \\
\hline Yes & 13 & 17 & 16 & 46 & $(36.8 \%)$ \\
No & 26 & 24 & 29 & 79 & $(63.2 \%)$ \\
\hline
\end{tabular}




\section{E. Questionnaire Page 4}

Q10: How old are you?

\begin{tabular}{lccccc}
\hline & WhatsApp & Skype & Facebook Messenger & \multicolumn{2}{c}{ All Conditions } \\
\hline$<\mathbf{2 0}$ & 6 & 10 & 9 & 25 & $(20.0 \%)$ \\
$\mathbf{2 0 - 3 4}$ & 32 & 26 & 30 & 88 & $(70.4 \%)$ \\
$\mathbf{3 5 - 4 9}$ & 1 & 3 & 4 & 8 & $(6.4 \%)$ \\
$\geq \mathbf{5 0}$ & 0 & 2 & 1 & 3 & $(2.4 \%)$ \\
No answer & 0 & 0 & 1 & 1 & $(0.8 \%)$ \\
\hline
\end{tabular}

Q11: With which gender do you identify?

\begin{tabular}{lccccr}
\hline & WhatsApp & Skype & Facebook Messenger & \multicolumn{2}{c}{ All Conditions } \\
\hline Female & 17 & 12 & 11 & 40 & $(32.0 \%)$ \\
Male & 21 & 28 & 31 & 80 & $(64.0 \%)$ \\
Other & 0 & 1 & 2 & 3 & $(2.4 \%)$ \\
No answer & 1 & 0 & 1 & 2 & $(1.6 \%)$ \\
\hline
\end{tabular}

Q12: Please estimate your level of experience with mobile devices. (1 - Beginner, 5 - Expert)

\begin{tabular}{lcccrr}
\hline & WhatsApp & Skype & Facebook Messenger & \multicolumn{2}{c}{ All Conditions } \\
\hline $\mathbf{1}$ (beginner) & 0 & 1 & 0 & 1 & $(0.8 \%)$ \\
$\mathbf{2}$ & 3 & 3 & 5 & 11 & $(8.8 \%)$ \\
$\mathbf{3}$ & 7 & 11 & 12 & 30 & $(24.0 \%)$ \\
$\mathbf{4}$ & 16 & 15 & 19 & 50 & $(40.0 \%)$ \\
$\mathbf{5}$ (expert) & 11 & 10 & 7 & 28 & $(22.4 \%)$ \\
No answer & 2 & 1 & 2 & 5 & $(4.0 \%)$ \\
\hline
\end{tabular}

\section{F. Questionnaire Page 5}

Q13: Does this result match your expectations?

\begin{tabular}{lccclc}
\hline & WhatsApp & Skype & Facebook Messenger & All Conditions \\
\hline Yes & 31 & 20 & 32 & 83 & $(66.4 \%)$ \\
No & 8 & 21 & 13 & 42 & $(33.6 \%)$ \\
\hline
\end{tabular}

Q14: Why does this result match your expectations? Why not?

- Free text

G. Questionnaire Page 6

Q15: Do you think the delete function should be limited (e.g., only messages of the last hour, only the latest message, only unread messages could be deleted)?

\begin{tabular}{lcccll}
\hline & WhatsApp & Skype & Facebook Messenger & \multicolumn{2}{l}{ All Conditions } \\
\hline Yes & 10 & 16 & 13 & 39 & $(31.2 \%)$ \\
No & 29 & 25 & 32 & 86 & $(68.8 \%)$ \\
\hline
\end{tabular}

Q16: How should the delete function be limited? Please specify.

- Free text 